\title{
Predictive value of a reduction in the level of high-density lipoprotein-cholesterol in patients with non-small-cell lung cancer undergoing radical resection and adjuvant chemotherapy: a retrospective observational study
}

Fan Luo ${ }^{1 \dagger}$, Kang-mei Zeng ${ }^{1 * \dagger}$, Jia-xin Cao ${ }^{1 \dagger}$, Ting Zhou ${ }^{1 \dagger}$, Su-xia Lin ${ }^{2}$, Wen-juan Ma ${ }^{1}$, Yun-peng Yang ${ }^{1}$, Zhong-han Zhang ${ }^{1}$, Fei-teng Lu' ${ }^{1}$ Yan Huang ${ }^{1}$, Hong-yun Zhao ${ }^{3^{*}}$ and Li Zhang ${ }^{1^{*}}$

\begin{abstract}
Background: Cancer patients often exhibit chemotherapy-associated changes in serum lipid profiles, however, their prognostic value before and after adjuvant chemotherapy on survival among non-small-cell lung cancer (NSCLC) patients is unknown.

Methods: NSCLC patients undergoing radical resection and subsequent adjuvant chemotherapy from 2013 to 2017 at Sun Yat-sen University Cancer Center were retrospectively reviewed. Fasted serum lipid levels were measured before and after chemotherapy. The optimal lipid cut-off values at baseline and fluctuation were determined using $X$-tile ${ }^{T M}$. The fluctuations in serum lipid levels and disease-free survival (DFS) were assessed.
\end{abstract}

\footnotetext{
* Correspondence: cengkm@sysucc.org.cn; zhaohy@sysucc.org.cn;

zhangli6@mail.sysu.edu.cn

${ }^{\dagger}$ Fan Luo, Kang-mei Zeng, Jia-xin Cao and Ting Zhou contributed equally to this work.

${ }^{1}$ State Key Laboratory of Oncology in South China, Collaborative Innovation Center for Cancer Medicine, Department of Medical Oncology, Guangdong Esophageal Cancer Institute, Sun Yat- sen University Cancer Center, 510060 Guangzhou, People's Republic of China

${ }^{3}$ State Key Laboratory of Oncology in South China, Collaborative Innovation Center for Cancer Medicine, Department of Clinical Research, Guangdong Esophageal Cancer Institute, Sun Yat- sen University Cancer Center, 510060 Guangzhou, People's Republic of China

Full list of author information is available at the end of the article
}

C C The Author(s). 2021 Open Access This article is licensed under a Creative Commons Attribution 4.0 International License, which permits use, sharing, adaptation, distribution and reproduction in any medium or format, as long as you give appropriate credit to the original author(s) and the source, provide a link to the Creative Commons licence, and indicate if changes were made. The images or other third party material in this article are included in the article's Creative Commons. licence, unless indicated otherwise in a credit line to the material. If material is not included in the article's Creative Commons licence and your intended use is not permitted by statutory regulation or exceeds the permitted use, you will need to obtain permission directly from the copyright holder. To view a copy of this licence, visit http://creativecommons.org/licenses/by/4.0/ The Creative Commons Public Domain Dedication waiver (http://creativecommons.org/publicdomain/zero/1.0/) applies to the data made available in this article, unless otherwise stated in a credit line to the data. 
Results: Serum cholesterol, low-density lipoprotein cholesterol (LDL-C), high-density lipoprotein-cholesterol (HDL-C), triglyceride, apolipoprotein (Apo) A-I, and ApoB all significantly increased after adjuvant chemotherapy. X-tile determined $1.52 \mathrm{mmol} / \mathrm{L}$ of HDL-C and $0.74 \mathrm{~g} / \mathrm{L}$ of $A p o B$ as the optimal cut-off values before chemotherapy. Patients with HDL-C $\geq 1.52 \mathrm{mmol} / \mathrm{L}$ (median DFS: not reached vs. 26.30 months, $P=0.0005$ ) and a decreased HDL-C level after adjuvant chemotherapy (median DFS: 80.43 vs. 26.12 months, $P=0.0204$ ) had a longer DFS. An HDL-C level that increased by $\geq 0.32 \mathrm{mmol} / \mathrm{L}$ after chemotherapy indicated a worse DFS. A high baseline ApoB level were associated with a superior DFS. In the univariate analysis and the multivariate Cox analyses, a high baseline HDL-C level and a HDL-C reduction after adjuvant chemotherapy were independent indicators for superior DFS. High baseline HDL-C was related to N0-1 stage $\left(X^{2}=6.413, P=0.011\right)$, and HDL-C fluctuation was significantly correlated with specific chemotherapy regimens $\left(X^{2}=5.002, P=0.025\right)$.

Conclusions: Adjuvant chemotherapy increased various lipid levels in resected NSCLC patients. A higher HDL-C level before chemotherapy and a reduced HDL-C level after adjuvant chemotherapy were independent predictors of longer DFS in patients with curable NSCLC.

Keywords: Non-small-cell lung cancer, Adjuvant chemotherapy, High-density lipoprotein-cholesterol, Disease-free survival, Prognosis

\section{Background}

Lung cancer (LC) is the most common cancer, with 80 $-85 \%$ of cases non-small-cell lung cancer (NSCLC) [1-3]. Almost one-third of NSCLC patients are candidates for resection $[4,5]$, and the number is increasing thanks to more and improved lung cancer screening [6]. However, even after curative resection, $10-75 \%$ of patients develop local or distant recurrence [7]. For stage II-IIIA patients undergoing radical resection, adjuvant chemotherapy is recommended as the standard of care to improve outcomes [8]. To date, the tumor, node, and metastasis staging system is the most discriminative indicator of prognostic value [7]. However, 5-year survival rates range from $73 \%$ for stage IA disease to $13 \%$ for stage IV disease, and even patients with the same stage can have heterogenous outcomes [7]. As such, more predictive methods are needed.

Several reports have demonstrated that aberrant metabolism of lipids can affect cancer development [9-11]. An altered serum lipid profile [cholesterol, low-density lipoprotein-cholesterol (LDL-C), highdensity lipoprotein-cholesterol (HDL-C), apolipoprotein (Apo) A-I (the major protein component of HDL-C), ApoB (the main protein component of LDL$\mathrm{C}$ ), and triglyceride (TG)] is often observed in cancer patients. Dysfunctional lipid metabolism in NSCLC has also been reported. The total level of cholesterol (particularly HDL-C) tends to be inversely linked to LC risk [12, 13]. Similarly, ApoA-I and ApoB play a role in LC development. The ApoA-I serum level is inversely correlated with an increased risk of $\mathrm{LC}$, whereas, ApoB is positively associated with LC incidence [14]. Whether the abnormal metabolism of lipids affects the prognosis of NSCLC patients receiving curative treatments is unknown.

Chemotherapy agents can also affect lipid metabolism. A few studies assessed breast cancer patients who received chemotherapy, and discovered significantly increased total cholesterol, LDL-C, and TG levels [15-18]. In colorectal cancer patients, chemotherapy significantly increased HDL-C and decreased LDL-C [19]. The molecular mechanisms underlying chemotherapy-related lipid imbalance are unclear. Additionally, cytotoxic drugs have been reported to influence system lipid metabolism by affecting cholesterol synthesis and efflux [20].

Chemotherapy-related lipid alteration can predict patients' outcomes. When patients with acute lymphoblastic leukemia (ALL) were treated with induction treatment, dynamic increases in serum HDL-C and ApoA1 predicted better chemotherapy efficacy [21]. For patients with small cell lung cancer treated with chemotherapy, elevation in serum LDL-C predicted poor survival [22]. Yet, whether fluctuations in lipid levels exist in patients with NSCLC undergoing adjuvant chemotherapy, and whether they can predict NSCLC prognosis is unknown.

This retrospective study aimed to assess disease-free survival (DFS) prediction using dynamic lipid changes after adjuvant chemotherapy for NSCLC patients undergoing curative resection.

\section{Methods \\ Patients}

The inclusion criteria were: (a) pathologically confirmed primary NSCLC stage IB-IIIA; (b) available clinical information (sex, age, height, weight, smoking history, drinking history, diabetes mellitus, hyperlipidemia, metabolic syndrome, hypertension comorbidities, and the use of lipid-affecting drugs); (c) underwent chemotherapy for four to six cycles in an adjuvant setting following radical resection; (d) age $\geq 18$ years old; (e) data available on fasted serum lipid profiles measured within 1 week 
before receiving the first chemotherapy cycle and between 2 to 4 weeks after the last chemotherapy treatment (cholesterol, TG, HDL-C, LDL-C, ApoA-I, and $\mathrm{ApoB}$ ); (f) normal liver function (alanine aminotransferase (ALT), aspartate aminotransferase (AST), and total bilirubin (T-BIL)) measured within 1 week before starting adjuvant treatment.

The exclusion criteria were: (a) neoadjuvant chemoradiotherapy; (b) concomitant tumors or malignant tumors diagnosed within 3 years of the LC diagnosis; (c) use of drugs affecting lipid metabolism before serum collection (mainly statins); (d) concomitant diseases associated with lipid metabolism-related disease, such as diabetes, hyperlipidemia, or metabolic syndrome; (e) clinical obesity, a $\mathrm{BMI} \geq 30 \mathrm{~kg} / \mathrm{m}^{2}$; and (f) concomitant hypertension.

Figure 1 shows the patient enrollment process. One hundred and seventeen patients were assessed with NSCLC who received curative-intent resection from 2013 to 2017 at Sun Yat-sen University Cancer Center (SYSUCC), Guangzhou, China. This study was conducted in accordance with the ethical standards of SYSUCC, and the Declaration of Helsinki (2013).

\section{$X$-tile ${ }^{\mathrm{TM}}$}

X-tile (Yale University School of Medicine, New Haven, CT, USA) offers a rigorous statistical estimation for dividing a cohort into a "low" or "high" group for lipid serum levels. For DFS, X-tile plots were used to evaluate the optimal cutoff values for serum lipids according Camp et al.'s method [23].

\section{Data collection}

As part of the physical examination, fasted serum lipid profiles were analyzed before and after adjuvant chemotherapy. Fasted blood specimens were collected before breakfast and tested using an automatic biochemical analyzer (Hitachi 7600-020, Japan).

Based on the medical records, demographic and clinical parameters (sex, age, height, weight, histology, smoking history, drinking history, diabetes mellitus, hyperlipidemia, metabolic syndrome, hypertension comorbidities, disease stage, radiotherapy, surgical type, adjuvant chemotherapy regimens, and use of lipidaffecting drugs) were collected. The tumor stage at the diagnosis of NSCLC was defined using the National Comprehensive Cancer Network guidelines.

The end-point of the study was DFS, which was defined as the period from surgery to the earliest radiographic evidence of recurrence, death, or the date of last patient access (censored). Radiological findings were confirmed by two experienced oncologists. Follow-up took place every 3 months for 2 years after surgery, and then every 6 months thereafter, or until death. The last follow-up was April 2020.

\section{Statistical analyses}

Statistical analyses were performed using SPSS 25 (IBM, Armonk, NY, USA). Conventional statistics were used to study any fluctuations in the lipid levels and the correlations with clinical factors. The optimal cutoffs for serum lipid profiles were determined using X-tile, which helped to stratify patients according to different outcomes. The association between serum lipids with DFS was determined using the Kaplan-Meier survival methods. Cox proportional hazards regression models were applied for the univariate and multivariate analyses. Risk factors in the univariate analyses with a $P$ value $<0.15$ were included in the multivariate analyses. Data were the mean \pm standard deviation (SD). Alterations in serum lipids and lipoproteins pre- and post-chemotherapy were compared using paired $t$-tests. A $P<0.05$ was considered significant.

\section{Results \\ Participants}

Table 1 describes the clinical characteristics of this study's cohort. The median follow-up was 58.8 months and patients were diagnosed at a median age of 56 (range, 31-70) years old. Most patients were male (69/ $117,59 \%)$. As for histology subtypes, lung adenocarcinoma accounted for $73.5 \%(86 / 117)$ of cases. Fifty-four (46.2\%) cases were stage IB-II disease, and 63 (53.8\%) were stage IIIA disease. The main surgery type was lobectomy (93.3\%). Chemotherapy regimens administered mainly included cisplatin/carboplatin/nedaplatin + pemetrexed (86/117, $73.5 \%)$. Other regimens were cisplatin/ carboplatin/nedaplatin + docetaxel $(14 / 117,12.0 \%)$, cisplatin/carboplatin/nedaplatin + paclitaxel (9/117, 7.7\%), and cisplatin + gemcitabine $(8 / 117,6.8 \%)$.

\section{Serum lipid and lipoprotein level comparisons before and after chemotherapy}

The serum lipid profile before chemotherapy and after chemotherapy completion were analyzed for each patient to reveal any associations. The level of cholesterol, TG, HDL-C, LDL-C, ApoA-I, and ApoB all significantly increased after adjuvant chemotherapy ( $P \leq 0.001$ for all) (Table 2). The mean HDL-C level pre- and postchemotherapy was $1.20 \mathrm{mmol} / \mathrm{L}$, and $1.44 \mathrm{mmol} / \mathrm{L}$.

\section{Serum lipid profile evaluation before chemotherapy using $X$-tile}

$\mathrm{X}$-tile was used to find the optimal cutoff value for the serum lipids based on DFS. The cutoff for the HDL-C serum level before adjuvant chemotherapy was 1.52 $\mathrm{mmol} / \mathrm{L}$. Using this value, patients were stratified as having a high risk of disease recurrence (Fig. 2A). The 


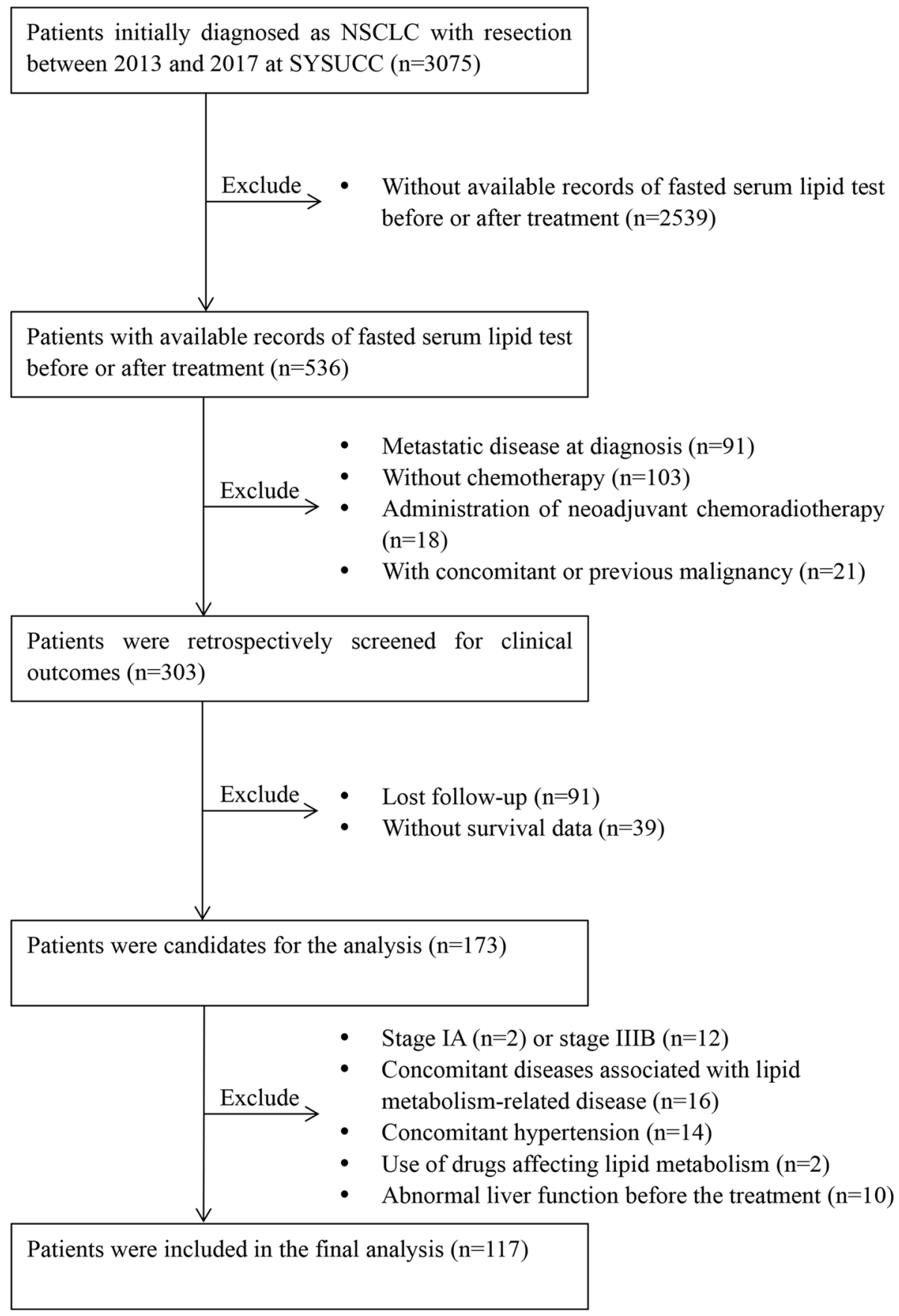

Fig. 1 Study flowchart

optimal cutoff value for cholesterol, TG, LDL-C, ApoA$\mathrm{I}$, and $\mathrm{ApoB}$, were $6.12 \mathrm{mmol} / \mathrm{L}, 1.36 \mathrm{mmol} / \mathrm{L}, 2.08$ $\mathrm{mmol} / \mathrm{L}, 0.85 \mathrm{~g} / \mathrm{L}$, and $0.74 \mathrm{~g} / \mathrm{L}$, respectively.

\section{Lipid-related survival estimates for DFS}

Serum lipid and lipoprotein levels before adjuvant chemotherapy were categorized as "high" or "low" based on the cut-offs determined by X-tile (Table 3). The relationship between the lipid levels and DFS for NSCLC patients were analyzed. The median DFS for patients with high HDL-C was unavailable, as only seven out of 24 patients reached the endpoints, while patients with low HDL-C had a median DFS of 26.30 months (range, 0.70-85.47). High baseline HDL-C was associated with a superior DFS $(P=0.0005)$ (Fig. $3 \mathrm{~A}$ and Table 3$)$. The median DFS for patients with high ApoB and those with low ApoB was 37.80 months and 11.83 months, respectively $(P=0.0316)$ (Table 3$)$. There were no statistical 
Table 1 Characteristics of all patients

\begin{tabular}{|c|c|c|}
\hline Characteristics & Cases $(n=117)$ & Percentage (\%) \\
\hline \multicolumn{3}{|l|}{ Age (years) } \\
\hline Median (range) & $56(31-70)$ & \\
\hline$<65$ vs. $\geq 65$ & 99 vs. 18 & 84.6 vs. 15.4 \\
\hline \multicolumn{3}{|l|}{ Gender } \\
\hline Male vs. female & 69 vs. 48 & 59 vs. 41 \\
\hline \multicolumn{3}{|l|}{ BMI } \\
\hline Median (range) & $22.23(16.42-29.39)$ & \\
\hline$<24 \mathrm{~kg} / \mathrm{m}^{2}$ vs. $\geq 24 \mathrm{~kg} / \mathrm{m}^{2}$ & 79 vs. 38 & 67.5 vs. 32.5 \\
\hline \multicolumn{3}{|l|}{ Smoking status } \\
\hline Non-smokers vs. smokers & 63 vs. 54 & 53.8 vs. 46.2 \\
\hline \multicolumn{3}{|l|}{ Drinking status } \\
\hline Non-drinkers vs. drinkers & 84 vs. 33 & 71.8 vs. 28.2 \\
\hline \multicolumn{3}{|l|}{ Histology } \\
\hline Adenocarcinoma vs. non-adenocarcinoma & 86 vs.31 & 73.5 vs. 26.5 \\
\hline \multicolumn{3}{|l|}{ T stage } \\
\hline $1-2$ & 93 & 79.5 \\
\hline $3-4$ & 24 & 20.5 \\
\hline \multicolumn{3}{|l|}{ N stage } \\
\hline $0-1$ & 62 & 53 \\
\hline 2 & 55 & 47 \\
\hline \multicolumn{3}{|l|}{ Disease stage } \\
\hline $\mid \mathrm{B}-\|$ & 54 & 46.2 \\
\hline$\| \mathrm{A}$ & 63 & 53.8 \\
\hline \multicolumn{3}{|l|}{ Surgery type } \\
\hline Lobectomy & 109 & 93.3 \\
\hline Pneumonectomy & 8 & 6.7 \\
\hline \multicolumn{3}{|l|}{ Radiotherapy } \\
\hline No & 106 & 90.6 \\
\hline Yes & 11 & 9.4 \\
\hline \multicolumn{3}{|l|}{ Adjuvant chemotherapy regimens } \\
\hline Cisplatin/carboplatin/nedaplatin + pemetrexed & 86 & 73.5 \\
\hline Others & 31 & 26.5 \\
\hline Cisplatin/carboplatin/nedaplatin + docetaxel & 14 & 12.0 \\
\hline Cisplatin/carboplatin/nedaplatin + paclitaxel & 9 & 7.7 \\
\hline Cisplatin + gemcitabine & 8 & 6.8 \\
\hline
\end{tabular}

Abbreviations: $T$ tumor, $N$ node, $B M I$ Body Mass Index

differences between the high and low cohort for cholesterol, triglyceride, LDL-C or ApoA-I.

Correlations between serum lipid fluctuations and survival were analyzed (Tables 4 and 5). Patients with a serum HDL-C level that decreased after adjuvant chemotherapy exhibited superior DFS compared with patients with an increased level $(P=0.0204)$. The median DFS for patients with elevated HDL-C levels was 26.12 (range, 0.70-85.47) months, while the median DFS for patients with decreased HDL-C levels was 80.43 (range, 10.53-81.17) months (Table 4, and Fig. 3B). Specifically, the optimal cut-off value of lipid fluctuation after adjuvant chemotherapy was determined using X-tile (Fig. 2B). The results suggested that NSCLC patients with a serum HDL-C level that increased by $0.32 \mathrm{mmol} / \mathrm{L}$ or above after chemotherapy had an inferior DFS (HDL-C fluctuation $<0.32$ vs. $\geq 0.32 \mathrm{mmol} / \mathrm{L}$ : median DFS: 49.70 vs. 19.17 months, $P=0.0069$ ) (Table 5, and Fig. 3C). 
Table 2 Lipid alterations in all patients

\begin{tabular}{|c|c|c|}
\hline Lipids & Mean \pm SD & $P$ value ${ }^{\phi}$ \\
\hline Cholesterol (mmol/L) & & $<0.001$ \\
\hline Pre-chemotherapy level & $4.73 \pm 1.02$ & \\
\hline Post-chemotherapy level & $5.51 \pm 1.00$ & \\
\hline Difference $^{a}$ & $0.78 \pm 0.96$ & \\
\hline Triglyceride (mmol/L) & & $<0.001$ \\
\hline Pre-chemotherapy level & $1.22 \pm 0.57$ & \\
\hline Post-chemotherapy level & $1.51 \pm 0.81$ & \\
\hline Difference $^{a}$ & $0.29 \pm 0.069$ & \\
\hline HDL-C (mmol/L) & & $<0.001$ \\
\hline Pre-chemotherapy level & $1.20 \pm 0.32$ & \\
\hline Post-chemotherapy level & $1.44 \pm 0.35$ & \\
\hline Difference $^{a}$ & $0.24 \pm 0.36$ & \\
\hline LDL-C (mmol/L) & & $<0.001$ \\
\hline Pre-chemotherapy level & $2.98 \pm 0.91$ & \\
\hline Post-chemotherapy level & $3.50 \pm 0.88$ & \\
\hline Difference $^{a}$ & $0.52 \pm 0.76$ & \\
\hline ApoA-I (g/L) & & $<0.001$ \\
\hline Pre-chemotherapy level & $1.17 \pm 0.28$ & \\
\hline Post-chemotherapy level & $1.47 \pm 0.27$ & \\
\hline Difference $^{a}$ & $0.30 \pm 0.30$ & \\
\hline ApoB (g/L) & & $<0.001$ \\
\hline Pre-chemotherapy level & $0.98 \pm 0.24$ & \\
\hline Post-chemotherapy level & $1.08 \pm 0.24$ & \\
\hline Difference $^{a}$ & $0.10 \pm 0.21$ & \\
\hline
\end{tabular}

Data are mean \pm standard deviations

Abbreviations: $S D$ standard deviations, $H D L-C$ high-density lipoprotein

cholesterol, LDL-C low-density lipoprotein cholesterol, ApoA-I apolipoprotein

A-I, ApoB apolipoprotein B

${ }^{\mathrm{a}}$ Difference $=$ lipids post-chemotherapy -lipids pre-chemotherapy

${ }^{\phi}$ Compared with paired t-test

Moreover, a triglyceride increase $\geq 0.25 \mathrm{mmol} / \mathrm{L}$ and an increase in ApoA-I $\geq 0.50 \mathrm{~g} / \mathrm{L}$ indicated a poorer DFS (Table 5).

\section{Prognostic indicators for NSCLC after chemotherapy}

Whether serum lipid and lipoprotein levels (or fluctuations in their levels) during chemotherapy could predict DFS was explored for stage IB-IIIA NSCLC patients undergoing radical resection and adjuvant chemotherapy. Serum lipid and lipoprotein levels before chemotherapy were classified as high or low, lipid profile chemotherapy-related fluctuations, and other clinicopathological characteristics were included in the univariate and multivariate analyses (Table 6).

In the univariate analyses, a chemotherapy-related HDL-C reduction exhibited superior DFS (hazard ratio [HR]: 0.475; $95 \%$ confidence interval [CI]: 0.249-0.904, $P=0.023)$. In the multivariate analyses, a chemotherapy- related HDL-C reduction independently predicted DFS (HR: 0.609; 95 \%CI: 0.310-1.200, $P=0.015$ ). A high baseline HDL-C level also independently indicated superior DFS (low vs. high: HR: 1.717; 95 \%CI: 0.735$4.014, P=0.021)$. N stage was found to be an inferior independent indicator for DFS. In the univariate analyses, $\mathrm{N}$ stage was associated with DFS (N0-1 vs. N2: HR: 0.329; 95 \%CI: $0.202-0.534, P<0.001)$. In the multivariate Cox regression analyses, $\mathrm{N}$ stage was predictive of DFS (N0-1 vs. N2: HR: 0.353; 95 \%CI: 0.203-0.612, $P<$ 0.001). And a low baseline ApoB and drinking had a negative effect on DFS in the univariate analysis (Table 6).

\section{Correlations between HDL-C and clinical characteristics}

A number of correlations between HDL-C and clinical characteristics were observed: (1) a high baseline HDL-C was related to N0-1 stage $\left(X^{2}=6.413, P=0.011\right)$, (2) non-smokers $\left(X^{2}=6.106, P=0.013\right)$ and chemotherapy other than pemetrexed-based regimens $\left(X^{2}=5.002, P=\right.$ 0.025 ) were prone to a HDL-C reduction, and (3) no association was found between HDL-C level and the other parameters, including gender, BMI, stage or surgical type (Table 7).

\section{Discussion}

Here, serum lipid profiles in predicting outcomes were assessed in NSCLC patients who received adjuvant chemotherapy after radical surgery. Cholesterol, LDL-C, HDL-C, ApoA-I, ApoB, and TG levels were all significantly elevated after chemotherapy. Patients with a high HDL-C, and ApoB at baseline as well as a chemotherapyrelated reduction in the $\mathrm{HDL}-\mathrm{C}$ level had a longer DFS. Furthermore, a reduction in the HDL-C level, and a high HDL-C level at baseline were independent predictors of a longer DFS.

Extensive studies have indicated that serum lipid levels are altered following chemotherapy. Yet, the evidence is inconsistent, with differences among various cancers. An in vitro study on the commonly used lung cancer drug docetaxel showed increased HDL-C biogenesis in primary human skin fibroblasts, macrophages and smooth muscle cells [24]. An earlier study on chemo-sensitive cancers also showed an increase in cholesterol and LDLC levels after chemotherapy [25].

However, LDL-C and HDL-C can also be altered in reverse. Wang's large cohort study of 667 colorectal cancer patients exhibited significantly increased HDL-C and decreased LDL-C after receiving fluoropyrimidine-based chemotherapy [19]. According to Li, and Arpino et al. patients with breast cancer showed significant LDL-C elevation and HDL-C reduction post-chemotherapy [15, 16]. Sharma $M$ et al. found that specific drugs also have different effects. Doxorubicin decreased HDL-C, 


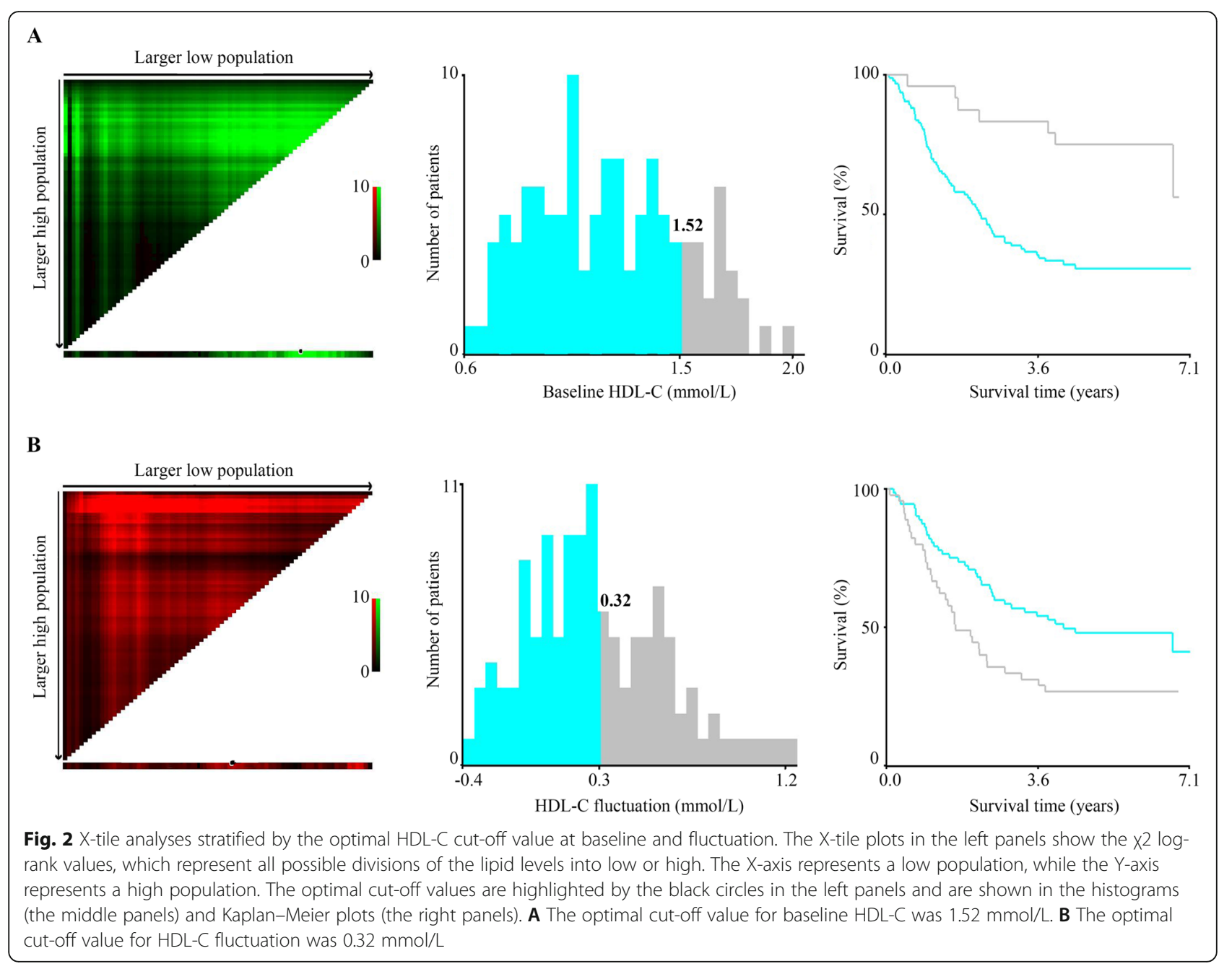

whereas, paclitaxel increased ApoB, yet cyclophosphamide had no effect on HDL metabolism in breast cancer patients [20]. Other agents, 5'-fluorouracil and methotrexate might also decrease HDL-C and LDL-C levels [26]. Nevertheless, this study here, is the first to report the lipid fluctuations after adjuvant chemotherapy in NSCLC. Pemetrexed-based regimens specifically, were more prone to increasing HDL-C.

There are fewer studies on chemotherapy affecting blood lipids, with most focusing on the mechanisms. Some chemotherapy drugs can affect lipid metabolism in liver cells. Doxorubicin decreases the expression of 3hydroxy-3-methylglutaryl-coenzymeA (HMG-CoA) reductase and ATP-binding cassette transporter A1 (ABCA1) in liver cells by directly decreasing two transcription factors, the peroxisomal proliferator activated receptor $\gamma($ PPAR $\gamma)$ and the liver $X$ receptor $\alpha(L X R \alpha)$ [20]. In liver cells, HMG-CoA reductase mediates cholesterol synthesis and ABCA1 mediates cholesterol efflux, which are major contributors to HDL-C levels [27]. However, the effect is drug-specific, for example, cyclophosphamide, and paclitaxel do not express the same activity [20]. It has also been discovered that chemotherapy can affect system lipid metabolism on primary human skin fibroblasts, macrophages and smooth muscle cells. Desmocollin 1 (DSC1) binds to apoA-I (apoA-I-DSC1) to inhibit HDL biogenesis, whereas, docetaxel binds with high affinity as a potential inhibitor of apoA-I-DSC1 interaction [24]. These varying results on chemotherapy-related lipid alterations are probably due to the multi-faceted mechanisms that vary by cancer type and drugs. In a word, chemotherapy agents do alter lipid levels, however, accurately predicting how those levels impact cancer prognosis differs greatly.

Gender, according to Tharu BP et al., is a confounding factor. The correlation with HDL-C and gender was also analyzed in this study. Neither the baseline HDL-C nor chemotherapy-related HDL-C fluctuations were significantly different between male and females. Similarly, no association between HDL-C and gender was found in patients with gallbladder cancer [28]. However, several studies have reported higher concentrations of HDL-C 
Table 3 Baseline lipids for DFS

\begin{tabular}{|c|c|c|c|c|}
\hline Lipids & N (\%) & Number of events & DFS (months), median and range & $P$ value \\
\hline Cholesterol $^{a}$ & & & & 0.132 \\
\hline High & $105(89.7)$ & 66 & $80.43(7.80-81.17)$ & \\
\hline Low & $12(10.3)$ & 5 & $29.33(0.70-85.47)$ & \\
\hline Triglyceride $^{a}$ & & & & 0.0529 \\
\hline High & $34(29.1)$ & 25 & $25.05(1.70-83.8)$ & \\
\hline Low & $83(70.9)$ & 46 & $44.50(0.70-85.47)$ & \\
\hline HDL-C ${ }^{\mathrm{a}}$ & & & & 0.0005 \\
\hline High & $24(20.5)$ & 7 & Unmature (5.83-82.13) & \\
\hline Low & $93(79.5)$ & 64 & $26.30(0.70-85.47)$ & \\
\hline LDL-C ${ }^{a}$ & & & & 0.0520 \\
\hline High & $101(86.3)$ & 59 & $37.80(0.70-85.47)$ & \\
\hline Low & $16(13.7)$ & 12 & $14.75(3.23-64.63)$ & \\
\hline ApoA-1 ${ }^{\mathrm{a}}$ & & & & 0.252 \\
\hline High & $102(87.2)$ & 65 & $30.20(0.70-82.33)$ & \\
\hline Low & $15(12.8)$ & 6 & Unmature (4.60-85.47) & \\
\hline$A_{p o B}{ }^{a}$ & & & & 0.0316 \\
\hline High & $103(88.0)$ & 61 & $37.80(0.70-85.47)$ & \\
\hline Low & $14(12.0)$ & 11 & $11.83(3.23-63.37)$ & \\
\hline
\end{tabular}

Abbreviations: DFS disease free survival, HDL-C high-density lipoprotein cholesterol, LDL-C low-density lipoprotein cholesterol, ApoA-I apolipoprotein A-I, $A p o B$ apolipoprotein B

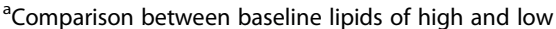

in women. One gastric cancer study indicated that males had significantly lower HDL-C levels [29]. This may be attributed to sex hormone differences.

In this study, both a high baseline HDL-C and chemotherapy-related HDL-C reduction were identified to independently predict DFS for curable patients with resected NSCLC. While HDL-C often exhibits an association with tumor incidence, the results are inconsistent. HDL-C was adversely associated with a risk of breast cancer and colon cancer [30-33] but it was reported that an increased HDL-C level is related to a high risk of non-Hodgkin lymphoma [34], and colorectal cancer
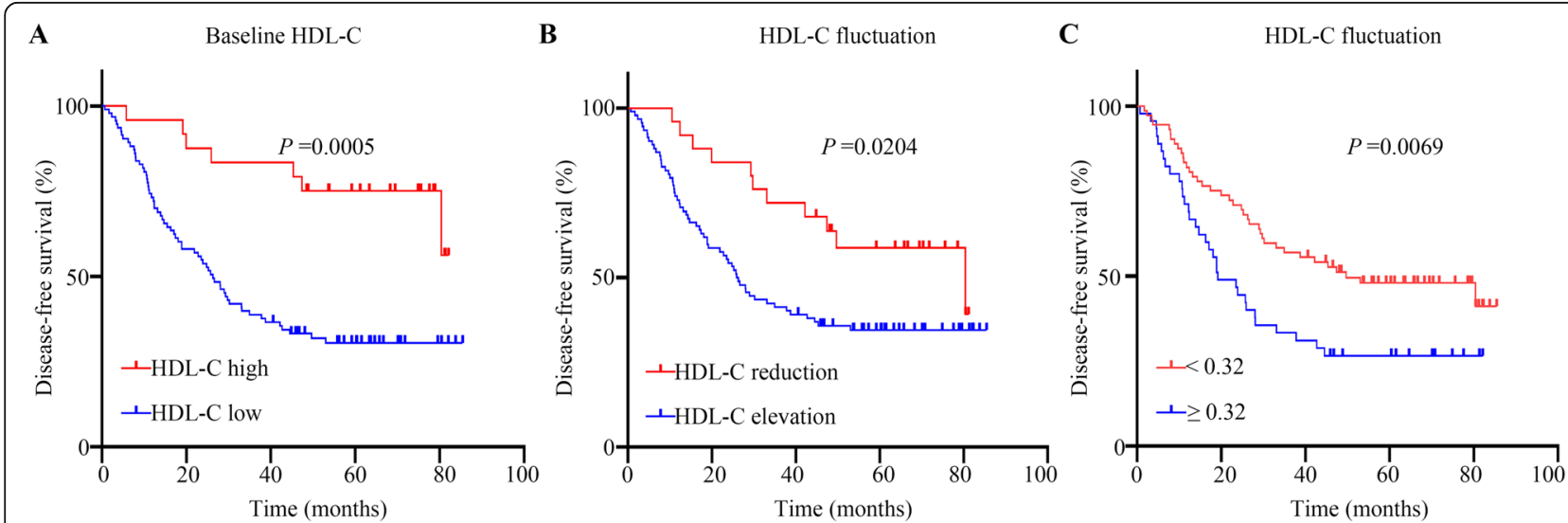

Fig. 3 Survival curves. A: DFS differed significantly between patients with a serum HDL-C level at baseline $\geq 1.52 \mathrm{mmol} / \mathrm{L}$ and those with a serum HDL-C level at baseline $<1.52 \mathrm{mmol} / \mathrm{L}$ (median DFS: not reached vs. 26.30 months, $P=0.0005$ ). B: DFS differed significantly between patients with an increased HDL-C and those with a decreased HDL-C after adjuvant chemotherapy compared with baseline (median DFS: 26.12 vs. 80.43 months, $P=0.0204)$. C: DFS differed significantly between patients with a serum HDL-C level that increased by $\geq 0.32 \mathrm{mmol} / \mathrm{L}$ and those with a serum HDL-C level that increased by $<0.32 \mathrm{mmol} / \mathrm{L}$ (median DFS: 19.17 vs. 49.70 months, $P=0.0069$ ) 
Table 4 Lipid alterations for DFS

\begin{tabular}{|c|c|c|c|c|}
\hline Lipids & N (\%) & Number of events & DFS (months), median and range & $P$ value \\
\hline Cholesterol $^{a}$ & & & & 0.775 \\
\hline Elevation & $93(79.5)$ & 55 & $33.10(1.70-85.47)$ & \\
\hline Reduction & $24(20.5)$ & 16 & $34.47(0.70-81.17)$ & \\
\hline Triglyceride $^{a}$ & & & & 0.949 \\
\hline Elevation & $82(70.1)$ & 50 & $34.03(0.70-85.47)$ & \\
\hline Reduction & $35(29.9)$ & 21 & $30.20(3.23-81.37)$ & \\
\hline HDL-C ${ }^{a}$ & & & & 0.0204 \\
\hline Elevation & $92(78.6)$ & 60 & $26.12(0.70-85.47)$ & \\
\hline Reduction & $25(21.4)$ & 11 & $80.43(10.53-81.17)$ & \\
\hline LDL-C ${ }^{a}$ & & & & 0.919 \\
\hline Elevation & $84(71.8)$ & 50 & $31.22(1.70-85.47)$ & \\
\hline Reduction & $33(28.2)$ & 21 & $34.97(0.70-81.17)$ & \\
\hline ApoA-1 ${ }^{a}$ & & & & 0.286 \\
\hline Elevation & $97(82.9)$ & 61 & $28.97(0.70-85.47)$ & \\
\hline Reduction & $20(17.1)$ & 10 & $80.43(1.70-81.17)$ & \\
\hline $\mathrm{ApoB}^{\mathrm{a}}$ & & & & 0.887 \\
\hline Elevation & $81(69.2)$ & 49 & $33.10(1.70-85.47)$ & \\
\hline Reduction & $36(30.8)$ & 22 & $34.47(0.70-81.17)$ & \\
\hline
\end{tabular}

Data are mean \pm standard deviations

Abbreviations: DFS disease free survival, HDL-C high-density lipoprotein cholesterol, LDL-C low-density lipoprotein cholesterol, ApoA-I apolipoprotein A-I, $A p o B$ apolipoprotein B

${ }^{a}$ Comparison between lipids post-chemotherapy and lipids pre-chemotherapy

Table 5 Optimal cut-off values of lipid fluctuation for DFS

\begin{tabular}{|c|c|c|c|c|}
\hline Lipid fluctuation $^{a}$ & $\mathrm{~N}(\%)$ & Number of events & DFS (months), median and range & $P$ value \\
\hline Cholesterol (mmol/L) & & & & 0.20 \\
\hline$<0.14$ & $16(13.7)$ & 8 & $80.43(0.70-81.17)$ & \\
\hline$\geq 0.14$ & $101(86.3)$ & 63 & $28.97(1.70-85.47)$ & \\
\hline Triglyceride $(\mathrm{mmol} / \mathrm{L})$ & & & & 0.020 \\
\hline$<0.25$ & $61(52.1)$ & 31 & $47.40(3.23-85.47)$ & \\
\hline$\geq 0.25$ & $56(47.9)$ & 40 & $26.28(0.70-83.80)$ & \\
\hline HDL-C (mmol/L) & & & & 0.0069 \\
\hline$<0.32$ & $72(61.5)$ & 38 & $49.70(1.70-85.47)$ & \\
\hline$\geq 0.32$ & $45(38.5)$ & 33 & $19.17(0.70-82.13)$ & \\
\hline LDL-C (mmol/L) & & & & 0.17 \\
\hline$<1.15$ & $91(77.8)$ & 59 & $29.73(0.70-82.33)$ & \\
\hline$\geq 1.15$ & $26(22.2)$ & 12 & Unmature (3.23-85.47) & \\
\hline ApoA-I (g/L) & & & & 0.018 \\
\hline$<0.50$ & $90(76.9)$ & 50 & $42.17(0.70-85.47)$ & \\
\hline$\geq 0.50$ & $27(23.1)$ & 21 & $18.93(4.60-77.60)$ & \\
\hline ApoB $(g / L)$ & & & & 0.14 \\
\hline$<0.14$ & $72(61.5)$ & 48 & $27.32(0.70-82.07)$ & \\
\hline$\geq 0.14$ & $45(38.5)$ & 23 & $53.00(2.37-85.47)$ & \\
\hline
\end{tabular}

Data are mean \pm standard deviations

Abbreviations: DFS disease free survival, HDL-C high-density lipoprotein cholesterol, LDL-C low-density lipoprotein cholesterol, ApoA-I apolipoprotein A-I, $A p o B$ apolipoprotein B

${ }^{\mathrm{a}}$ Difference $=$ lipids post-chemotherapy -lipids pre-chemotherapy 
Table 6 Predictive factors for DFS by univariate and multivariate analysis

\begin{tabular}{|c|c|c|c|c|c|}
\hline & & \multicolumn{2}{|l|}{ Univariate analyses } & \multicolumn{2}{|c|}{ Multivariate analyses } \\
\hline & & $\mathrm{HR}(95 \% \mathrm{CI})$ & Pvalue & $\mathrm{HR}(95 \% \mathrm{Cl})$ & Pvalue \\
\hline Gender & male vs. female & $0.954(0.594-1.533)$ & 0.847 & & \\
\hline Year & $<65$ vs. $\geq 65$ & $1.035(0.740-1.447)$ & 0.842 & & \\
\hline BMI $\left(\mathrm{kg} / \mathrm{m}^{2}\right)$ & $<24$ vs. $\geq 24$ & $1.026(0.624-1.699)$ & 0.918 & & \\
\hline Histology & adenocarcinoma vs. non-adenocarcinoma & $0.944(0.553-1.612)$ & 0.833 & & \\
\hline Disease Stage & |-II vs. I|| & $1.011(0.633-1.613)$ & 0.965 & & \\
\hline Smoking status & smokers vs. non-smokers & $1.656(1.022-2.685)$ & 0.041 & $1.025(0.575-1.830)$ & 0.932 \\
\hline Drinking status & non-drinkers vs. drinkers & $1.219(0.705-2.106)$ & 0.478 & & \\
\hline Surgical type & lobectomy vs. pneumonectomy & $2.062(0.648-6.564)$ & 0.221 & & \\
\hline T stage & $1-2$ vs. $3-4$ & $0.567(0.311-1.035)$ & 0.054 & $0.719(0.384-1.345)$ & 0.302 \\
\hline $\mathrm{N}$ stage & $0-1$ vs. 2 & $0.329(0.202-0.534)$ & $<0.001$ & $0.353(0.203-0.612)$ & $<0.001$ \\
\hline Radiotherapy & no vs. yes & $0.727(0.348-1.521)$ & 0.398 & & \\
\hline Baseline cholesterol & low vs. high & 1.986 (0.799-4.938) & 0.140 & $1.449(0.508-4.130)$ & 0.488 \\
\hline Baseline triglyceride & low vs. high & $0.620(0.380-1.011)$ & 0.055 & $0.652(0.385-1.103)$ & 0.111 \\
\hline Baseline HDL-C & low vs. high & $2.702(1.291-5.652)$ & 0.008 & $1.717(0.735-4.014)$ & 0.021 \\
\hline Baseline LDL-C & low vs. high & $1.837(0.985-3.427)$ & 0.056 & $0.803(0.386-1.671)$ & 0.557 \\
\hline Baseline ApoA-I & low vs. high & $0.616(0.266-1.423)$ & 0.256 & & \\
\hline Baseline ApoB & low vs. high & $2.047(1.073-3.905)$ & 0.030 & $2.100(0.999-4.417)$ & 0.050 \\
\hline Cholesterol fluctuation & reduction vs. elevation & $1.085(0.622-1.893)$ & 0.775 & & \\
\hline Triglyceride fluctuation & reduction vs. elevation & $0.984(0.591-1.638)$ & 0.949 & & \\
\hline HDL-C fluctuation & reduction vs. elevation & $0.475(0.249-0.904)$ & 0.023 & $0.609(0.310-1.200)$ & 0.015 \\
\hline LDL-C fluctuation & reduction vs. elevation & $1.027(0.616-1.711)$ & 0.919 & & \\
\hline ApoA-I fluctuation & reduction vs. elevation & $0.696(0.356-1.359)$ & 0.289 & & \\
\hline ApoB fluctuation & reduction vs. elevation & $1.037(0.627-1.717)$ & 0.887 & & \\
\hline
\end{tabular}

Data are mean \pm standard deviations

Abbreviations: DFS disease free survival, BMI Body Mass Index, HDL-C high-density lipoprotein cholesterol, $L D L-C$ low-density lipoprotein cholesterol, ApoA-I apolipoprotein A-I, $A p o B$ apolipoprotein B, HR Hazard ratio, Cl Confidence interval

[19]. A lower HDL-C serum level at baseline has also been associated with tumor progression and an increase mortality among various cancers [35-37]. As for the relationship between chemotherapy-related HDL-C and tumors, patients with a high level of HDL-C before chemotherapy were reported to have a better treatment response for breast cancer [38], gastric cancer [35], and prostate cancer [39]. In breast cancer, LDL-C elevation after chemotherapy showed better clinical responses [38]. Patients with a complete remission exhibited a significant HDL-C elevation after induction treatment for ALL [21]. For small cell lung cancer patients, chemotherapy with a decreased HDL-C may predict disease progression [22], yet, chemotherapy with an elevated HDL-C indicated a better survival for colorectal cancer patients [19].

Evidence concerning the possible mechanism for the favorable influence of HDL-C on DFS have been examined in some experimental studies where HDL-C was used to induce apoptosis, generate oxidative stress, and dysregulate the inflammation system and thereby, influence carcinogenesis $[40,41]$. The NSCLC patients in this study received surgical resection, and so the immune system may play an important part in the elimination of potential residual micro-metastases. The anti-tumor effect of HDL-C both in humans and in vivo are associated with altered immune responses [42, 43]. Speculatively, chemotherapy-related HDL-C and survival may be explained by the interaction between the cancer type, chemotherapy regimen and the immune system. However, the role of HDL-C in immunomodulation has both defensive and inflammatory effects. In cancers, HDL-C can regulate anti-tumor immunity and increase the recruitment of cytotoxic $\mathrm{T}$ cells, decrease the recruitment of myeloid-derived suppressor cells and promote the accumulation of M1 macrophages into the tumor environment $[43,44]$. HDL-C has also been found to mediate an immune-suppressive effect in some non-cancerous 
Table 7 Relationships between HDL-C and clinical data

\begin{tabular}{|c|c|c|c|c|c|c|}
\hline \multirow[b]{2}{*}{ Characteristics } & \multicolumn{3}{|c|}{ Baseline HDL-C ${ }^{a}$} & \multicolumn{3}{|c|}{ HDL-C fluctuation ${ }^{b}$} \\
\hline & High & Low & $P$ Value & Elevation & Reduction & $P$ Value \\
\hline \multicolumn{7}{|l|}{ Age, n (\%) } \\
\hline$<65$ & $16(13.7)$ & $83(70.9)$ & 0.086 & $77(65.8)$ & $22(18.8)$ & 0.597 \\
\hline$\geq 65$ & $6(5.1)$ & $12(10.3)$ & & $15(12.8)$ & $3(2.6)$ & \\
\hline \multicolumn{7}{|l|}{ Gender, n (\%) } \\
\hline Female & $11(9.4)$ & $36(30.8)$ & 0.297 & $40(34.2)$ & $7(6.0)$ & 0.162 \\
\hline Male & $11(9.4)$ & $59(50.4)$ & & $52(44.4)$ & $18(15.4)$ & \\
\hline \multicolumn{7}{|l|}{ BMI $\left(k g / m^{2}\right), n(\%)$} \\
\hline$<24$ & $16(13.7)$ & $63(53.8)$ & 0.563 & $60(51.3)$ & $19(16.2)$ & 0.307 \\
\hline$\geq 24$ & $6(5.1)$ & $32(27.4)$ & & $32(27.4)$ & $6(5.1)$ & \\
\hline \multicolumn{7}{|l|}{ Smoking status, n (\%) } \\
\hline Non-smoker & $8(6.8)$ & $55(47.0)$ & 0.068 & $55(47.0)$ & $8(6.8)$ & 0.013 \\
\hline Smoker & $14(12.0)$ & $40(34.2)$ & & 37 (31.6) & $17(14.5)$ & \\
\hline \multicolumn{7}{|l|}{ T stage, n (\%) } \\
\hline $1-2$ & $19(16.2)$ & $74(63.2)$ & 0.375 & $73(62.4)$ & $20(17.1)$ & 0.943 \\
\hline $3-4$ & $3(2.7)$ & $21(17.9)$ & & $19(16.2)$ & $5(4.3)$ & \\
\hline \multicolumn{7}{|l|}{ N stage, n (\%) } \\
\hline $0-1$ & $17(14.5)$ & $45(38.5)$ & 0.011 & $45(38.5)$ & $17(14.5)$ & 0.090 \\
\hline 2 & $5(4.3)$ & $50(42.7)$ & & $47(40.2)$ & $8(6.8)$ & \\
\hline \multicolumn{7}{|l|}{ Disease stage, n (\%) } \\
\hline Stage I-II & $8(6.8)$ & $45(38.5)$ & 0.350 & $41(35.0)$ & $12(10.3)$ & 0.760 \\
\hline Stage III & $14(12.0)$ & $50(42.7)$ & & $51(43.6)$ & $13(11.1)$ & \\
\hline \multicolumn{7}{|l|}{ Histology, n (\%) } \\
\hline Adenocarcinoma & $15(12.8)$ & $71(60.7)$ & 0.530 & $67(57.3)$ & $19(16.2)$ & 0.750 \\
\hline Non-adenocarcinoma & $7(6.0)$ & $24(20.5)$ & & $25(21.4)$ & $6(5.1)$ & \\
\hline \multicolumn{7}{|l|}{ Drinking status, n (\%) } \\
\hline Non-drinkers & $14(12.0)$ & $71(60.7)$ & 0.293 & $68(58.1)$ & $17(14.5)$ & 0.556 \\
\hline Drinkers & $8(6.8)$ & $24(20.5)$ & & $24(20.5)$ & $8(6.8)$ & \\
\hline \multicolumn{7}{|l|}{ Radiotherapy, n (\%) } \\
\hline Non-radiotherapy & $21(17.9)$ & $85(72.6)$ & 0.386 & $83(70.9)$ & $23(19.7)$ & 0.787 \\
\hline Radiotherapy & $1(1.0)$ & $10(8.5)$ & & $9(7.7)$ & $2(1.7)$ & \\
\hline \multicolumn{7}{|l|}{ chemotherapy regimens, $\mathrm{n}(\%)$} \\
\hline Cisplatin/carboplatin/nedaplatin + pemetrexed & $15(12.8)$ & $71(60.7)$ & 0.530 & $72(61.5)$ & $14(12.0)$ & 0.025 \\
\hline Others & $7(6.0)$ & $24(20.5)$ & & $20(17.1)$ & $11(9.4)$ & \\
\hline \multicolumn{7}{|l|}{ Surgery type, n (\%) } \\
\hline Lobectomy & $22(18.8)$ & $87(74.4)$ & 0.158 & $85(72.6)$ & $24(20.5)$ & 0.526 \\
\hline Pneumonectomy & $0(0)$ & $8(6.8)$ & & $7(6.0)$ & $1(0.9)$ & \\
\hline
\end{tabular}

Abbreviations: HDL-C high-density lipoprotein cholesterol, BMI Body Mass Index

${ }^{a}$ Comparison between baseline lipids of high and low

${ }^{\mathrm{b}}$ Comparison between lipids post-chemotherapy and lipids pre-chemotherapy

diseases. HDL-C increased $\mathrm{T}$ regulatory cells to attenuate the inflammatory process by promoting the free cholesterol efflux [45], and in vitro, HDL-C promoted an anti-inflammatory M2 phenotype [46]. M2 macrophages may stimulate tumor relapse after chemotherapy [47], with a lower level been shown to predict better prognosis for pancreatic cancer patients after chemotherapy [48].

As the recommended adjuvant treatment for NSCLC patients is cisplatin or carboplatin, it may increase M2 
macrophages induced by IL10 secreted from tumor cells [49]. It is speculated that a reduction in the HDL-C level after chemotherapy might reverse the chemotherapyinduced shift of M2 macrophages. This may explain why a reduction in the HDL-C level after chemotherapy is significantly correlated with longer DFS. However, further studies are needed to clarify the specific molecular mechanism between chemotherapy-related HDL-C and survival.

\section{Comparisons with other studies and what does the current work add to the existing knowledge}

Lipids are involved in tumor growth, immunomodulation and treatment response. Serum lipid levels also change during anti-cancer therapy. It was reported that cytotoxic drugs affect blood lipid metabolism differently [20]. Breast cancer patients often display increased total cholesterol, LDL-C, and TG levels during chemotherapy [15-18], while colorectal cancer patients treated with chemotherapy show increased HDL-C and decreased LDL-C [19]. However, it remains unclear whether lipid alterations exist in NSCLC patients receiving standard adjuvant chemotherapy. Here, elevation in lipid profiles was observed during adjuvant chemotherapy in patients with NSCLC. Moreover, associations between fluctuating lipid levels and the risk of cancer progression have been studied in only a few cancers including gastric cancer, ovarian cancer and small-cell lung cancer $[19,22,36$, 37]. One common observation among these cancers was that a lower HDL-C serum level at baseline was associated with an increase in cancer progression and mortality [22, 35-37], yet, colorectal cancer patients showed a superior outcome with a chemotherapy-related elevated HDL-C [19]. The associations between fluctuating serum lipids and the clinical outcome of curable NSCLC are unknown.

In this study, relationships between chemotherapyrelated lipid alterations and the survival of NSCLC patients were identified. Notably, this is the first study concerning chemotherapy-associated blood lipid fluctuations and its relationship with clinical outcomes for NSCLC patients. According to this study, a lower baseline HDL level and an increased HDL level after adjuvant chemotherapy are independent prognostic indicators for disease-free survival in NSCLC patients. In support of these findings, a recent exploratory analysis of 851 cases with colorectal cancer demonstrated that chemotherapy-related HDL-C elevation was determined to be an independent prognostic indicator for superior DFS [19]. Specifically, this study determined $0.32 \mathrm{mmol} / \mathrm{L}$ as the optimal cut-off value for HDL-C fluctuation after chemotherapy, with an HDL-C fluctuation $<0.32 \mathrm{mmol} / \mathrm{L}$ indicating a better DFS. No previous study has reported a specific lipid fluctuation cut-off value for the survival of cancer patients. Compared with other available diagnostic methods, the serum HDL test is fast, repeatable, inexpensive, and simple to carry out, and it would enable more patients to monitor recurrence. These results hint that simple HDL-C fluctuation may be a useful future prognostic marker for NSCLC.

\section{Strengths and limitations}

This is the first study to investigate the association between serum lipids and DFS in NSCLC patients treated by radical resection and adjuvant chemotherapy. The potential bias caused by the confounding factors were minimized, which improved the reliability of the findings. However, there are some limitations: (1) it was a retrospective study; (2) patients were pooled from a single center; (3) the study cohort was small; (4) we did not have OS data; and (5) lipid and lipoprotein levels were stratified in a simple dichotomous manner, more precise stratification might have affected the prognostic efficacy.

\section{Conclusions}

This is the first study to determine the relationship between serum lipid profiles and DFS in NSCLC patients treated by radical resection and adjuvant chemotherapy. Chemotherapy significantly increased the levels of various lipids and lipoproteins. Patients with a higher level at baseline of HDL-C and ApoB as well as a reduction in the HDL-C level after chemotherapy tended to have a longer DFS. Serum HDL-C levels that increased by 0.32 $\mathrm{mmol} / \mathrm{L}$ or above after chemotherapy had an inferior DFS. A chemotherapy-associated reduction in HDL-C was a favorable prognostic indicator for DFS, and may have predictive value in an adjuvant setting. Therefore, the monitoring and regulation of serum lipid profiles are of great value for lung cancer patients receiving adjuvant chemotherapy. Clinically, it may be possible to predict survival by observing fluctuations in serum lipid levels. In the future, it is anticipated that it will be a reliable indicator in predicting lung cancer prognosis. This study suggests that serum lipids should be under long-term monitoring for cancer management.

\section{Abbreviations \\ LC: Lung cancer; NSCLC: Non-small-cell lung cancer; LDL-C: Low-density lipoprotein-cholesterol; HDL-C: High-density lipoprotein-cholesterol; Apo: Apolipoprotein; TG: Triglyceride; DFS: Disease-free survival; ALT: Alanine aminotransferase; AST: Aspartate aminotransferase; T-BIL: Total bilirubin; ALL: Acute lymphoblastic leukemia; SYSUCC: Sun Yat-sen University Cancer Center; SD: Standard deviation; HR: Hazard ratio; Cl: Confidence interval; HMG-CoA: 3-hydroxy-3-methylglutaryl-coenzymeA; ABCA1: ATP-binding cassette transporter A1; PPARY: Peroxisomal proliferator activated receptor $\gamma$; LXRa: Liver X receptor a; DSC1: Desmocollin 1}

\section{Acknowledgements}

Not applicable.

\section{Authors' contributions}

(I) Conception and design: HY Zhao, L Zhang; (II) administrative support: HY Zhao; (III) provision of study materials and patients: FT Lu, JX Cao; (IV) 
collection and data assembly: F Luo, KM Zeng, JX Cao, T Zhou; (V) data analysis and interpretation: F Luo, KM Zeng, T Zhou, JX Cao; (VI) manuscript writing: all authors; (VII) final approval of manuscript: all authors.

\section{Funding}

Sources of support: this work was supported by grants from the 5010 Clinical Research Foundation of Sun Yat-sen University (2016001); the Natural Science Foundation of Guangdong Province of China (2018A0303130243,

2020A1515011464); the Chinese National Natural Science Foundation project (82073396, 81872201); the Science and Technology Program of Guangdong (2017B020227001); the Chinese National Natural Science Foundation project (81872499); the Medical Scientific Research Foundation of Guangdong Province; China (C2018062); the Chinese National Natural Science Foundation project (82002628); and the China Postdoctoral Science Foundation (2019M660227)

\section{Availability of data and materials}

The datasets used and analysed during the current study are available from the corresponding author on reasonable request. The authenticity of this article has been validated with key raw data uploaded onto the Research Data Deposit (RDD)public platform (https:// www. researchdata. org. cn). The approval RDD number is RDDA2021002116.

\section{Declarations}

\section{Ethics approval and consent to participate}

This study was deemed exempt from institutional review board approval by Sun Yat-sen University Cancer Center (ethics approval number: B2021-05601). This study was conducted in accordance with the ethical standards of the institutional research committee and the Declaration of Helsinki (as revised in 2013). Due to the retrospective nature of this research, the requirement for informed consent was waived.

\section{Consent for publication}

Not applicable.

\section{Competing interests}

The authors declare that they have no competing interests.

\section{Author details}

${ }^{1}$ State Key Laboratory of Oncology in South China, Collaborative Innovation Center for Cancer Medicine, Department of Medical Oncology, Guangdong Esophageal Cancer Institute, Sun Yat- sen University Cancer Center, 510060 Guangzhou, People's Republic of China. ${ }^{2}$ State Key Laboratory of Oncology in South China, Collaborative Innovation Center for Cancer Medicine, Department of Pathology, Guangdong Esophageal Cancer Institute, Sun Yat-sen University Cancer Center, 510060 Guangzhou, People's Republic of China. ${ }^{3}$ State Key Laboratory of Oncology in South China, Collaborative Innovation Center for Cancer Medicine, Department of Clinical Research, Guangdong Esophageal Cancer Institute, Sun Yat- sen University Cancer Center, 510060 Guangzhou, People's Republic of China.

\section{Received: 7 June 2021 Accepted: 31 August 2021}

\section{Published online: 20 September 2021}

\section{References}

1. Siegel RL, Miller KD, Jemal A. Cancer statistics, 2020. CA Cancer J Clin. 2020; 70:7-30. https://doi.org/10.3322/caac.21590.

2. Chen W, Zheng R, Baade PD, Zhang S, Zeng H, Bray F, Jemal A, Yu XQ, He J. Cancer statistics in China, 2015. CA Cancer J Clin. 2016;66:115-32. https:// doi.org/10.3322/caac.21338.

3. Chen W, Zheng R, Zeng H, Zhang S. Epidemiology of lung cancer in China. Thorac Cancer. 2015;6:209-15. https://doi.org/10.1111/1759-7714.12169.

4. Torre LA, Siegel RL, Jemal A. Lung cancer statistics. Adv Exp Med Biol. 2016; 893:1-19. https://doi.org/10.1007/978-3-319-24223-1_1.

5. Little AG, Gay EG, Gaspar LE, Stewart AK. National survey of non-small cell lung cancer in the United States: epidemiology, pathology and patterns of care. Lung Cancer. 2007;57:253-60. https://doi.org/10.1016/j.lungcan.2007.03.012

6. National Lung Screening Trial, Research T, Aberle DR, Adams AM, Berg CD, Black WC, Clapp JD, Fagerstrom RM, Gareen IF, Gatsonis C, Marcus PM, Sicks JD. Reduced lung-cancer mortality with low-dose computed tomographic screening. N Engl J Med. 2011;365:395-409. https://doi.org/10.1056/ NEJMoa1102873.

7. Goldstraw P, Chansky K, Crowley J, Rami-Porta R, Asamura H, Eberhardt WEE, Nicholson AG, Groome P, Mitchell A, Bolejack V, et al. The IASLC Lung Cancer Staging Project: Proposals for Revision of the TNM Stage Groupings in the Forthcoming (Eighth) Edition of the TNM Classification for Lung Cancer. J Thorac Oncol. 2016;1 1:39-51. https://doi.org/10.1016/j.jtho.2015.09.009.

8. Pisters KMW, Evans WK, Azzoli CG, Kris MG, Smith CA, Desch CE, Somerfield MR, Brouwers MC, Darling G, Ellis PM, et al. Cancer Care Ontario and American Society of Clinical Oncology adjuvant chemotherapy and adjuvant radiation therapy for stages I-IIIA resectable non small-cell lung cancer guideline. J Clin Oncol. 2007;25:5506-18. https://doi.org/10.1200/ JCO.2007.14.1226.

9. Muntoni S, Atzori L, Mereu R, Satta G, Macis MD, Congia M, Tedde A, Desogus A, Muntoni S. Serum lipoproteins and cancer. Nutr Metab Cardiovasc Dis. 2009; 19:218-25. https://doi.org/10.1016/j.numecd.2008.06.002.

10. Munir R, Usman H, Hasnain S, Smans K, Kalbacher H, Zaidi N. Atypical plasma lipid profile in cancer patients: cause or consequence? Biochimie. 2014;102:9-18. https://doi.org/10.1016/j.biochi.2014.03.010.

11. Vilchez JA, Martinez-Ruiz A, Sancho-Rodriguez N, Martinez-Hernandez P, Noguera-Velasco JA. The real role of prediagnostic high-density lipoprotein cholesterol and the cancer risk: a concise review. Eur J Clin Invest. 2014;44: 103-14. https://doi.org/10.1111/eci.12185.

12. Chandler PD, Song Y, Lin J, Zhang S, Sesso HD, Mora S, Giovannucci EL, Rexrode KE, Moorthy MV, Li C, et al. Lipid biomarkers and long-term risk of cancer in the Women's Health Study. Am J Clin Nutr. 2016;103:1397-407. https://doi.org/10.3945/ajcn.115.124321.

13. Jafri $H$, Alsheikh-Ali AA, Karas $\mathrm{RH}$. Baseline and on-treatment high-density lipoprotein cholesterol and the risk of cancer in randomized controlled trials of lipid-altering therapy. J Am Coll Cardiol. 2010;55:2846-54. https://doi. org/10.1016/j.jacc.2009.12.069.

14. Borgquist S, Butt T, Almgren P, Shiffman D, Stocks T, Orho-Melander M, Manjer J, Melander O. Apolipoproteins, lipids and risk of cancer. Int J Cancer. 2016;138:2648-56. https://doi.org/10.1002/ijc.30013.

15. Li X, Liu Z-I, Wu Y-t, Wu H, Dai W, Arshad B, Xu Z, Li H, Wu K, Kong LQ. Status of lipid and lipoprotein in female breast cancer patients at initial diagnosis and during chemotherapy. Lipids in Health Disease. 2018;17:91. https://doi.org/10.1186/s12944-018-0745-1.

16. Arpino G, De Angelis C, Buono G, Colao A, Giuliano M, Malgieri S, Cicala S, De Laurentiis M, Accurso A, Crispo A, et al. Metabolic and anthropometric changes in early breast cancer patients receiving adjuvant therapy. Breast Cancer Res Treat. 2015;154:127-32. https://doi.org/10.1007/s10549-015-3586-x.

17. Dieli-Conwright CM, Wong L, Waliany S, Bernstein L, Salehian B, Mortimer JE. An observational study to examine changes in metabolic syndrome components in patients with breast cancer receiving neoadjuvant or adjuvant chemotherapy. Cancer. 2016;122:2646-53. https://doi.org/10.1002/ cncr.30104.

18. He T, Wang C, Tan Q, Wang Z, Li J, Chen T, Cui K, Wu Y, Sun J, Zheng D, et al. Adjuvant chemotherapy-associated lipid changes in breast cancer patients: a real-word retrospective analysis. Medicine. 2020;99:e21498. https://doi.org/10.1097/md.0000000000021498.

19. Wang Y, Wang ZQ, Wang FH, Lei XF, Yan SM, Wang DS, Zhang F, Xu RH, Wang LY, Li YH. Predictive value of chemotherapy-related high-density lipoprotein cholesterol (HDL) elevation in patients with colorectal cancer receiving adjuvant chemotherapy: an exploratory analysis of 851 cases. Oncotarget. 2016;7:57290-300. https://doi.org/10.18632/oncotarget.10145.

20. Sharma M, Tuaine J, McLaren B, Waters DL, Black K, Jones LM, McCormick SP. Chemotherapy agents alter plasma lipids in breast cancer patients and show differential effects on lipid metabolism genes in liver cells. PLoS One. 2016;11:e0148049. https://doi.org/10.1371/journal.pone.0148049.

21. Baroni S, Scribano D, Pagano L, Zuppi C, Leone G, Giardina B. Lipids and lipoproteins in acute lymphoblastic leukaemia (ALL). Leuk Res. 1994;18:6434. https://doi.org/10.1016/0145-2126(94)90047-7.

22. Liu T, Zhou T, Luo F, Yang Y, Zhao S, Huang Y, Zhao H, Zhang L, Zhao Y. Clinical significance of kinetics of low-density lipoprotein cholesterol and its prognostic value in limited stage small cell lung cancer patients. Cancer Control. 2021; 28. 10732748211028257https://doi.org/10.1177/10732748211 028257.

23. Camp RL, Dolled-Filhart M, Rimm DL. X-tile: a new bio-informatics tool for biomarker assessment and outcome-based cut-point optimization. Clin Cancer Res. 2004;10:7252-9. https://doi.org/10.1158/1078-0432.CCR-04-0713. 
24. Choi HY, Ruel I, Genest J. Identification of docetaxel as a potential drug to promote HDL biogenesis. Front Pharmacol. 2021;12:679456. https://doi.org/1 $0.3389 /$ fphar.2021.679456.

25. Alexopoulos CG, Pournaras S, Vaslamatzis M, Avgerinos A, Raptis S. Changes in serum lipids and lipoproteins in cancer patients during chemotherapy. Cancer Chemother Pharmacol. 1992;30:412-6. https://doi.org/10.1007/ BF00689971.

26. Rzymowska J. Effect of cytotoxic chemotherapy on serum lipid levels in breast cancer patients. Pathobiology. 1999;67:129-32. https://doi.org/10.11 59/000028062.

27. Liang $\mathrm{K}$, Kim CH, Vaziri ND. HMG-CoA reductase inhibition reverses LCAT and $L D L$ receptor deficiencies and improves $H D L$ in rats with chronic renal failure. Am J Physiol Renal Physiol. 2005;288:F539-44. https://doi.org/10.11 52/ajprenal.00074.2004.

28. Yuan B, Fu J, Yu WL, Fu XH, Qiu YH, Yin L, Zhu B, Zhang YJ. Prognostic value of serum high-density lipoprotein cholesterol in patients with gallbladder cancer. Rev Esp Enferm Dig. 2019;111:839-45. https://doi.org/1 0.17235/reed.2019.6201/2019.

29. Pih GY, Gong EJ, Choi JY, Kim MJ, Ahn JY, Choe J, Bae SE, Chang HS, Na HK, Lee $\mathrm{JH}$, et al. Associations of serum lipid level with gastric cancer risk, pathology, and prognosis. Cancer Res Treat. 2021;53:445-56. https://doi. org/10.4143/crt.2020.599.

30. Høyer AP, Engholm G. Serum lipids and breast cancer risk: a cohort study of 5,207 Danish women. Cancer Causes Control. 1992:3:403-8. https://doi.org/1 $0.1007 / \mathrm{bf00051352.}$

31. Furberg AS, Veierød MB, Wilsgaard T, Bernstein L, Thune I. Serum highdensity lipoprotein cholesterol, metabolic profile, and breast cancer risk. J Natl Cancer Inst. 2004;96:1152-60. https://doi.org/10.1093/jnci/djh216.

32. Kucharska-Newton AM, Rosamond WD, Mink PJ, Alberg AJ, Shahar E, Folsom AR. HDL-cholesterol and incidence of breast cancer in the ARIC cohort study. Ann Epidemiol. 2008;18:671-7. https://doi.org/10.1016/j.a nnepidem.2008.06.006

33. Michalaki V, Koutroulis G, Syrigos K, Piperi C, Kalofoutis A. Evaluation of serum lipids and high-density lipoprotein subfractions (HDL2, HDL3) in postmenopausal patients with breast cancer. Mol Cell Biochem. 2005;268: 19-24. https://doi.org/10.1007/s11010-005-2993-4.

34. Lim U, Gayles T, Katki HA, Stolzenberg-Solomon R, Weinstein SJ, Pietinen P, Taylor PR, Virtamo J, Albanes D. Serum high-density lipoprotein cholesterol and risk of non-hodgkin lymphoma. Cancer Res. 2007;67:5569-74. https:// doi.org/10.1158/0008-5472.CAN-07-0212.

35. Tamura T, Inagawa S, Hisakura K, Enomoto T, Ohkohchi N. Evaluation of serum high-density lipoprotein cholesterol levels as a prognostic factor in gastric cancer patients. J Gastroenterol Hepatol. 2012;27:1635-40. https:// doi.org/10.1111/j.1440-1746.2012.07189.x.

36. Zhong G-C, Huang S-Q, Peng Y, Wan L, Wu Y-Q-L, Hu T-Y, Hu J-J, Hao F-B. $\mathrm{HDL}-\mathrm{C}$ is associated with mortality from all causes, cardiovascular disease and cancer in a J-shaped dose-response fashion: a pooled analysis of 37 prospective cohort studies. Eur J Prev Cardiol. 2020;27:1187-203. https://doi. org/10.1177/2047487320914756.

37. Ke C, Li A, Hou Y, Sun M, Yang K, Cheng J, Wang J, Ge T, Zhang F, Li Q, et al. Metabolic phenotyping for monitoring ovarian cancer patients. Sci Rep. 2016;6:23334. https://doi.org/10.1038/srep23334.

38. Qu F, Chen R, Peng Y, Ye Y, Tang Z, Wang Y, Zong B, Yu H, Liu S. Assessment of the predictive role of serum lipid profiles in breast cancer patients receiving neoadjuvant chemotherapy. J Breast Cancer. 2020;23:24658. https://doi.org/10.4048/jbc.2020.23.e32.

39. His M, Zelek L, Deschasaux M, Pouchieu C, Kesse-Guyot E, Hercberg S, Galan P, Latino-Martel P, Blacher J, Touvier M. Prospective associations between serum biomarkers of lipid metabolism and overall, breast and prostate cancer risk. Eur J Epidemiol. 2014;29:119-32. https://doi.org/10.1007/s10654014-9884-5.

40. Soran H, Hama S, Yadav R, Durrington PN. HDL functionality. Curr Opin Lipidol. 2012;23:353-66. https://doi.org/10.1097/MOL.0b013e328355ca25.

41. von Eckardstein $A$, Hersberger $M$, Rohrer L. Current understanding of the metabolism and biological actions of HDL. Curr Opin Clin Nutr Metab Care. 2005;8:147-52. https://doi.org/10.1097/00075197-200503000-00007.

42. Wang $Y$, Sun $X Q$, Lin HC, Wang DS, Wang ZQ, Shao Q, Wang FH, Yan SM, Liang JY, Zeng ZL, et al. Correlation between immune signature and highdensity lipoprotein cholesterol level in stage I//II colorectal cancer. Cancer Med. 2019;8:1209-17. https://doi.org/10.1002/cam4.1987.
43. Zamanian-Daryoush M, Lindner D, Tallant TC, Wang Z, Buffa J, Klipfell E, Parker Y, Hatala D, Parsons-Wingerter P, Rayman P, et al. The cardioprotective protein apolipoprotein A1 promotes potent antitumorigenic effects. J Biol Chem. 2013;288:21237-52. https://doi.org/10.1 074/jbc.M113.468967.

44. Zamanian-Daryoush M, DiDonato JA, Apolipoprotein A-I. Cancer. Front Pharmacol. 2015;6:265. https://doi.org/10.3389/fphar.2015.00265.

45. Sorci-Thomas MG, Thomas MJ. High density lipoprotein biogenesis, cholesterol efflux, and immune cell function. Arterioscler Thromb Vasc Biol. 2012;32:2561-5. https://doi.org/10.1161/ATVBAHA.112.300135.

46. Trakaki A, Marsche G. Current Understanding of the immunomodulatory activities of high-density lipoproteins. Biomedicines. 2021;9. https://doi.org/1 0.3390/biomedicines9060587.

47. Hughes R, Qian BZ, Rowan C, Muthana M, Keklikoglou I, Olson OC, Tazzyman S, Danson S, Addison C, Clemons M, et al. Perivascular M2 macrophages stimulate tumor relapse after chemotherapy. Cancer Res. 2015;75:3479-91. https://doi.org/10.1158/0008-5472.Can-14-3587.

48. Di Caro G, Cortese N, Castino GF, Grizzi F, Gavazzi F, Ridolfi C, Capretti G, Mineri R, Todoric J, Zerbi A, et al. Dual prognostic significance of tumourassociated macrophages in human pancreatic adenocarcinoma treated or untreated with chemotherapy. Gut. 2016;65:1710-20. https://doi.org/10.113 6/gutjnl-2015-309193.

49. Dijkgraaf EM, Heusinkveld M, Tummers B, Vogelpoel LT, Goedemans R, Jha V, Nortier JW, Welters MJ, Kroep JR, van der Burg SH. Chemotherapy alters monocyte differentiation to favor generation of cancer-supporting M2 macrophages in the tumor microenvironment. Cancer Res. 2013;73:2480-92. https://doi.org/10.1158/0008-5472.Can-12-3542.

\section{Publisher's Note}

Springer Nature remains neutral with regard to jurisdictional claims in published maps and institutional affiliations.
Ready to submit your research? Choose BMC and benefit from:

- fast, convenient online submission

- thorough peer review by experienced researchers in your field

- rapid publication on acceptance

- support for research data, including large and complex data types

- gold Open Access which fosters wider collaboration and increased citations

- maximum visibility for your research: over $100 \mathrm{M}$ website views per year

At BMC, research is always in progress.

Learn more biomedcentral.com/submissions 